This is an electronic reprint of the original article. This reprint may differ from the original in pagination and typographic detail.

Author(s): Mansfeld, D.; Izotov, I.; Skalyga, V.; Tarvainen, Olli; Kalvas, Taneli; Koivisto, Hannu; Komppula, Jani; Kronholm, Risto; Laulainen, Janne

Title: $\quad$ Dynamic regimes of cyclotron instability in the afterglow mode of minimum-B electron cyclotron resonance ion source plasma

Year: $\quad 2016$

Version:

Please cite the original version:

Mansfeld, D., Izotov, I., Skalyga, V., Tarvainen, O., Kalvas, T., Koivisto, H., Komppula, J., Kronholm, R., \& Laulainen, J. (2016). Dynamic regimes of cyclotron instability in the afterglow mode of minimum-B electron cyclotron resonance ion source plasma. Plasma Physics and Controlled Fusion, 58(4), Article 045019.

https://doi.org/10.1088/0741-3335/58/4/045019

All material supplied via JYX is protected by copyright and other intellectual property rights, and duplication or sale of all or part of any of the repository collections is not permitted, except that material may be duplicated by you for your research use or educational purposes in electronic or print form. You must obtain permission for any other use. Electronic or print copies may not be offered, whether for sale or otherwise to anyone who is not an authorised user. 


\title{
Dynamic regimes of cyclotron instability in the afterglow mode of minimum-B electron cyclotron resonance ion source plasma
}

D. Mansfeld ${ }^{1}$, I.Izotov ${ }^{1,2}$, V.Skalyga ${ }^{1,2}$, O.Tarvainen ${ }^{3}$, T. Kalvas ${ }^{3}$,H.Koivisto ${ }^{3}$, J.Komppula ${ }^{3}$, R. Kronholm ${ }^{3}$, J. Laulainen ${ }^{3}$

1 Institute of Applied Physics of Russian Academy of Sciences, 46 Ulyanova st., Nizhny Novgorod, Russia. 2 2 Lobachevsky State University of Nizhny Novgorod (UNN), 23 Gagarina st., Nizhny Novgorod, Russia. 3 University of Jyväskylä, Department of Physics, Jyväskylä, Finland.

\begin{abstract}
The paper is concerned with the dynamic regimes of cyclotron instabilities in nonequilibrium plasma of a minimum-B electron cyclotron resonance ion source operated in pulsed mode. The instability appears in decaying ion source plasma shortly (1 - $10 \mathrm{~ms})$ after switching off the microwave radiation of the klystron, and manifests itself in the form of powerful pulses of electromagnetic emission associated with precipitation of high energy electrons along the magnetic field lines. Recently it was shown that such plasma instability causes perturbations of the extracted ion current, which limit the performance of the ion source and generates strong bursts of bremsstrahlung emission. In this article we present time-resolved diagnostics of electromagnetic emission bursts related to cyclotron instability in the decaying plasma. The temporal resolution is sufficient to study the fine structure of the dynamic spectra of the electromagnetic emission at different operating regimes of the ion source. It was found that at different values of magnetic field and heating power the dynamic spectra demonstrate common features: decreasing frequency from burst to burst and always falling tone during a single burst of instability. The analysis has shown that the instability is driven by the resonant interaction of hot electrons, distributed between the ECR zone and the trap center, with slow extraordinary wave propagating quasi-parallel with respect to the external magnetic field.
\end{abstract}

\section{Introduction}

Microwave plasma discharges are extensively used in various branches of plasma technology, such as thin-film deposition, plasma etching, surface ion treatment and sputtering, sources of positive and negative ion beams, etc. A significant part of such discharges operate at low gas pressure with the plasma being heated through electron cyclotron resonance (ECR) and confined magnetically, e.g. in toroidal devices [1] and gasdynamic traps [2]. Electromagnetic emission from ECR-heated ITER-like high temperature plasmas, is used for imaging system with spatial resolution [3], for example. 
A specific feature of ECR heating is that the energy of the external source is mostly embedded into transverse momentum of the electrons, thereby forming positive gradient in the electron velocity distribution. Resonant interaction between energetic strongly anisotropic electrons and the electromagnetic waves makes the plasma prone to kinetic cyclotron instabilities, which manifest themselves as a generation of powerful electromagnetic radiation and bursts of energetic electrons from the magnetic trap. Studies of cyclotron instabilities in magnetically trapped laboratory plasmas have a long history but they still remain topical, mostly with the advent of powerful sources of microwave radiation (especially, gyrotrons), which allow sufficient increase of the energy input into the plasma, thereby increasing the energies and anisotropy of the non-equilibrium resonant particles. Cyclotron instability is an important channel for the loss of the excess energy stored by the electrons [4], hence limiting the range of achievable plasma parameters. For example, in modern ECR sources, which are widely used as injectors of heavy multi-charged ions into accelerators, the precipitation of particles due to instabilities significantly modify the energy distribution function of the hot electrons and charge state distribution of the ions, thus limiting the average charge state of extracted ion beams [5].

This paper is devoted to studying the dynamic regimes of cyclotron instabilities in a nonequilibrium plasma of a minimum-B electron cyclotron resonance ion source operated in pulsed mode. The production of intense ion beams in so-called "afterglow" mode is of significant interest since it allows achieving pulsed ion currents substantially higher than those during continuous ( $\mathrm{cw}$ ) operation [6,7]. It is worth noting that all heavy ion experiments at the Large Hadron Collider (LHC) at CERN are based on ion beams $\left(\mathrm{Pb}^{27+}\right)$ extracted from an ECR ion source operating in the afterglow mode [8]. The afterglow occurs when the microwave generator is switched off and the plasma begins to decay. The confinement time of cold, collisional electrons is shorter than the confinement time of relativistic hot electrons. Thus, the anisotropy of the EVDF increases during the afterglow transient. The energy, which is stored mostly by hot electrons [9], is rapidly released in the form of particle losses and electromagnetic radiation in optical, microwave, and $\mathrm{x}$-ray bands. Various types of instabilities can arise during the plasma decay, causing undesirable perturbations of the plasma parameters. The observed oscillations of extracted ion current during the plasma decay were recently associated with a cyclotron instability driven by the resonant interaction of plasma waves with hot electron component of the anisotropic velocity distribution [10]. In this article we study the fine structure of the dynamic spectra of electromagnetic emission bursts related to the cyclotron instability in decaying plasma of a minimum-B magnetic field configuration (ECR ion source). The research results can be also applied in understanding the primary mechanisms of cyclotron instabilities of the same origin, e.g. the most powerful electromagnetic emission in magnetosphere of the Earth (auroral kilometric radiations [11], VLF-ELF emission) and other planets (e.g. Jovian decametric radiation [12]).

Bursts of energetic electrons and electromagnetic emission related to cyclotron instabilities of extraordinary waves propagating in perpendicular direction to the magnetic field, were studied earlier in a decaying plasma of an ECR discharge confined in a mirror axisymmetric magnetic trap [13, 14]. The nonlinear instability growth phase was explained in terms of a cyclotron maser model [15]. A new source of powerful THz radiation (based on the model) in which the plasma serves as a non-linear trigger element was then proposed [16]. A new 
regime of electron cyclotron instability aimed at explaining the complex temporal patterns of detected electromagnetic radiation observed in decaying plasma was discussed in [17].

Detailed investigations of temporal frequency characteristics of the electromagnetic radiation generated during the plasma relaxation in an adiabatic magnetic trap have shown that the cyclotron instability occurs near the electron cyclotron frequency or its second harmonic [18]. The experimental challenge is that the dynamic spectra of short (tens of nanoseconds) electromagnetic pulses is rather wide, because the resonant electrons do not have a monoenergetic distribution and the region of wave-particle interaction is usually spread in non-uniform magnetic field, which affects the local relativistic electron cyclotron frequency. Studying the fine-structure of dynamic spectra of the electromagnetic emission has become possible only recently with the advent of methods for measuring the electromagnetic field with high temporal resolution. First experimental data on the dynamic spectra of five different types of kinetic instabilities in various conditions of ECR discharge plasma were obtained using this method [19]. The analysis of recorded electromagnetic spectra presented in this paper allowed us to determine the most probable electromagnetic mode which is excited due to instability in minimum-B field configuration.

\section{Experimental setup}

The experimental data were taken with the room-temperature A-ECR-U type JYFL $14 \mathrm{GHz}$ ECRIS [20]. A schematic figure of the ECRIS and the experimental setup is shown in Fig. 1. The plasma was heated by 100-600 $\mathrm{W}$ of microwave power of a klystron amplifier at the frequency of $14 \mathrm{GHz}$. The klystron was operated in a pulsed mode by controlling the lowpower input signal from an oscillator with a fast RF-switch. The magnetic field of the ion source is generated by two solenoid coils and a permanent magnet sextupole resulting to a socalled minimum-B field configuration. The minimum value of the magnetic field $\left(\mathrm{B}_{\min }\right)$ is achieved on the axis of the ion source in between the solenoid coils in axial direction. On the other hand the resonance condition for (non-relativistic) electron heating, i.e. $2 \pi f_{\mu w}=\frac{e B_{E C R}}{m c}$ ( $f_{\mu w}$ is the heating frequency, $\mathrm{c}-$ speed of light in vacuum, $e$ and $m$ are the electron charge and mass, respectively), is satisfied on a closed (nearly) ellipsoidal surface with constant magnetic field of $\mathrm{B}_{\mathrm{ECR}}=0.5 \mathrm{~T}$. The magnetic field strength can be adjusted by varying the solenoid coil currents, which affects the injection and extraction mirror ratios as well as the $B_{\text {min }} / B_{E C R}$. The ion source was operated in the range of $0.68<\mathrm{B}_{\min } / \mathrm{B}_{\mathrm{ECR}}<0.81$. The strength of the sextupole field on the plasma chamber wall at the magnetic pole is $1.07 \mathrm{~T}$ when the solenoids are not energized [21]. A 3D plot of the magnetic field lines depicting the position of the resonance surface is shown in Fig. 2a, while Fig. 2b shows the magnetic field strength on axis. A complete description of the magnetic field profile can be found from Ref. [10].

Argon plasmas in the pressure range of $1.2 \cdot 10^{-7} \div 1.6 \cdot 10^{-6}$ mbar were studied. The pressure readings were measured outside the plasma chamber with an ionization gauge connected to a radial diagnostics port of the ion source.

The electron cyclotron instabilities were detected with a Bismuth Germanate (BGO) scintillator coupled with a Na-doped CsI photomultiplier tube (PMT) with $<4 \mu$ s resolution as 
described in previous work [5]. The detector is sensitive to bursts of bremsstrahlung, generated at the wall of the plasma chamber by energetic electrons, which are expelled from the magnetic trap as a result of the instability.

Plasma microwave emission was measured through a WR-75 waveguide port incorporated into the injection iron plug, and normally used for injection of microwave power at secondary frequency in two-frequency heating mode. The emitted microwave signal was guided into Tektronix MSO 72504 DX oscilloscope through WR-75 waveguide, high voltage break, waveguide-to-coaxial transition, power limiter and tunable attenuator. The features of the oscilloscope - $100 \mathrm{Gs} / \mathrm{s}$ sampling rate and $25 \mathrm{GHz}$ bandwidth - allowed direct recording of the waveforms of electromagnetic field emitted by the plasma with temporal resolution of 20 ps. The frequency response of the WR-75 waveguide and waveguide-to-coaxial transition was measured to be flat in the range of $8-15 \mathrm{GHz}$. The WR62 port was used for launching the $14 \mathrm{GHz}$ microwave radiation.

The investigations were performed in decaying plasma after switching off the microwave signal klystron. The trailing edge of the TTL signal controlling the RF-switch was used to trigger the oscilloscope for high sampling rate acquisition of the microwave signal waveform.

\section{Experimental results}

Figure 3 presents typical waveforms of the microwave electric field (upper curve) and bremsstrahlung (lower curve) signals recorded during the plasma decay. The data were taken in afterglow of a plasma discharge, initially sustained by $600 \mathrm{~W}$ of injected microwave power at $2.1 \cdot 10^{-7}$ mbar of argon and $\mathrm{B}_{\min } / \mathrm{B}_{\mathrm{ECR}}=0.75$. The microwave bursts usually appear in $1-4$ $\mathrm{ms}$ after the $14 \mathrm{GHz}$ microwave signal is switched off, and consist of a sequence of pulses lasting typically 0.1-1.5 $\mu$ s with intervals of 1-10 ms. The bremsstrahlung power flux signal, which is attributed to the precipitation of hot electrons from the trap, consists of a sequence of peaks lasting for some tens of $\mu \mathrm{s}$. Although the temporal resolution of the current-mode Xray detector is not sufficient to detect variations of the bremsstrahlung signal at the level of $100 \mathrm{~ns}$, it is evident that the leading edge of each X-ray pulse is perfectly coincident with the microwave emission packets.

Figure 4 shows dynamic spectrograms of the microwave bursts, subsequently emitted during the plasma decay at $1.2 \mathrm{~ms}$ (fig. 4a) $2.96 \mathrm{~ms}$ (fig 4b ) $9.62 \mathrm{~ms}$ (fig 4c) following the trailing edge of the $14 \mathrm{GHz}$ microwave pulse. The signal was treated with Goertzel algorithm to build the spectral power density in each time domain in the range of 8-15 GHz with $10 \mathrm{MHz}$ step (the spectral power density of the signal is indicated with false color). The horizontal line at $12.50 \mathrm{GHz}$ is an artefact related to the sampling rate of the oscilloscope and subsequent analysis of the data. The emission of the first pulse starts at the frequency of $12.2 \mathrm{GHz}$ and ends after $1 \mu \mathrm{s}$ at $10.95 \mathrm{GHz}$, the second pulse lasts for $0.35 \mu$ s with the frequency decreasing from $11.25 \mathrm{GHz}$ to $10.3 \mathrm{GHz}$. The third, $0.1 \mu$ s pulse starts at $10.3 \mathrm{GHz}$ and ends at 9.7 $\mathrm{GHz}$. In other words, the frequency range of each wave packet is consistently lower in comparison to the preceding one. The dynamic spectrum of each pulse is typically 
descending in frequency, whereas the rate of frequency drift is increased with each consecutive pulse. However, in some cases the last pulse in the described sequence exhibits quasi-monochromatic narrow-banded harmonics shown in figure 5.

The range of emission frequencies $(12.2-9.7 \mathrm{GHz})$ during the plasma decay is comparable to the range of electron cyclotron frequencies between $\mathrm{B}_{\mathrm{ECR}}(0.5 \mathrm{~T})$ and $\mathrm{B}_{\min }(0.41 \mathrm{~T})$ at the trap center i.e. 14-11.5 GHz. It is worth noting that a significant part of the microwave signal is emitted at frequencies below the cyclotron frequency at the trap center $(11.5 \mathrm{GHz})$, which indicates that the wave-particle interaction can occur at Doppler shifted relativistic cyclotron resonance $\left(\omega=\omega_{c e} \sqrt{1-\frac{v_{h o t}^{2}}{c^{2}}}-k_{\|} v_{\|}\right.$, where $v_{h o t}=\sqrt{v_{\|}^{2}+v_{\perp}^{2}}$ is the velocity of the hot electrons, $v_{\|}$and $v_{\perp}$ are the longitudinal and transversal (with respect to the magnetic field) velocity components and $k_{\|}$is the longitudinal wavenumber.

The maximum amplitude of the microwave signal varies from 60 to $100 \mathrm{mV}$ with $-30 \mathrm{~dB}$ attenuation. Taking into account the input impedance of $50 \mathrm{Ohm}$ yields $70-200 \mathrm{~mW}$ peak power for the measured instability signals coupled to the waveguide. Unfortunately, the frequency-dependent coupling efficiency into the WR75-port, as well as the total power reflected from the plasma are unknown, so only very rough estimates of emitted power can be made. Assuming that the radiation is emitted into 4pi solid angle and is measured at distance of $\sim 10 \mathrm{~cm}$ i.e. the distance between the trap center and the entrance to the WR-75 waveguide, the upper limit of the microwave power can be estimated to be 70-200W.

It was found that at different ion source settings (B-field strength, neutral gas pressure and 14 $\mathrm{GHz}$ microwave power) the dynamic spectra demonstrate certain common features: (i) decreasing frequency from burst to burst and (ii) falling tone during a single instability burst. The magnetic field strength and heating power significantly affect the delay between switching off the klystron power and the appearance of the first instability pulse as well as the number of instability events during the afterglow. The dependence of the delay between switching off the klystron and the appearance of the instability on magnetic field strength and plasma heating power are shown in figs. $6 \mathrm{a}$ and $6 \mathrm{~b}$. The delay between the microwave switch off and the first instability event decreases monotonically with increasing magnetic field strength, while the dependence on heating power shows threshold-like dynamics being rather insensitive to the power above $300 \mathrm{~W}$. These observations can be explained by the fact that the instability growth rate depends on the density and anisotropy of the hot electrons, which are greatly increased at low gradients of magnetic field (corresponding to high $B_{\min } / B_{E C R}$ ) and high power as discussed in Ref. [10].

\section{Discussion}

The observed precipitation of energetic electrons from the trap and the generation of microwave bursts are inherently related to the excitation of electromagnetic waves due to cyclotron instabilities. The electron energy distribution function in steady state of ECRIS plasmas is considered to consist of three main fractions: cold (non-relativistic) electrons with an average energy $\left\langle E_{\text {cold }}\right\rangle$ of $10-100 \mathrm{eV}$, warm electrons with $\left\langle E_{\text {warm }}\right\rangle$ of $1-10 \mathrm{keV}$ and hot 
electrons with $\left\langle E_{\text {hot }}\right\rangle>10 \mathrm{keV}$ up to $1 \mathrm{MeV}[22,23]$. The dense (cold and warm) plasma component determines the dispersion relation and damping of propagating waves which can resonantly interact with the energetic electrons. Kinetic cyclotron instabilities are driven by the warm and hot electrons with an anisotropic velocity distribution in which the transverse velocity (with respect to the external magnetic field) is considerably higher than the longitudinal velocity. The interaction between the high-frequency waves and resonant electrons leads to rapid diffusion of energetic electrons in the velocity space. These electrons are eventually expelled into the loss cone and precipitated from the trap.

The most important plasma parameter which greatly affects the dispersion and polarization properties of the excited waves is the plasma density of cold and warm electrons. The density was not measured in the present afterglow experiment but can be estimated from well-known parameters of steady-state ECRIS plasma discharge. Experiments [22, 24] and simulations $[25,26]$ on ECRIS plasmas sustained by $14-18 \mathrm{GHz}$ microwave radiation imply that their electron density, $\mathrm{n}_{\mathrm{e}}$, is below $10^{12} \mathrm{~cm}^{-3}$ as a maximum i.e. well below the critical density of $2.4 \times 10^{12} \mathrm{~cm}^{-3}$. Since the electron plasma frequency, $f_{p e}$, is proportional to the electron density as $f_{p e} \propto n_{e}^{1 / 2}$, the maximum plasma frequency can be expected to be on the order of 9 $\mathrm{GHz}$ in the steady state. As soon as the microwave power is switched off the plasma begins to decay with different lifetimes for cold, warm and hot electron components. Cold electrons have the shortest confinement time of some tens of microseconds and rapidly leave the magnetic trap long before the appearance of the instabilities. The warm electron component has a characteristic lifetime of 1 to $20 \mathrm{~ms}$ as indicated by bremsstrahlung measurements [27] and, thus, does not decay completely by the onset of the instability, thereby determining the propagation of excited waves in the plasma. The hot electrons are well-confined in the magnetic trap for more than a second [28] and are believed to be responsible for the instabilities. The hot electrons are bouncing between the mirror points inside the magnetic

trap implying that the maximum anisotropy $\left(\mathrm{v}_{\perp}>\mathrm{v}_{\|}\right)$of the EVDF is achieved at their reflection points resulting in enhanced amplification of the excited wave at these locations. Let us define the mode of the excited wave in the afterglow using the experimental data together with the parameters of the ECRIS plasma and taking into account the dispersion relation for the warm electron component. We can neglect the contribution of thermal effects if not considering the exact cyclotron resonance condition i.e. $\left(k_{\|} v_{\|}<<\omega-\omega_{c e}\right)$. The most evident influence of thermal corrections can be observed in the shift of cut-off point of the dispersion relation, which allows the fast extraordinary waves to escape from the rarefied plasma [29]. Being quite typical for space plasma this specific case could hardly be realized in the present experimental conditions.

The range of (cold) electron gyrofrequencies, $f_{c e}=\omega_{c e} / 2 \pi$, is defined by the magnetic field configuration. Instabilities are observed when $\mathrm{B}_{\min } / \mathrm{B}_{\mathrm{ECR}}>0.75$, i.e. $\mathrm{B}_{\min }>0.375 \mathrm{~T}$, which implies that $f_{c e}>10.5 \mathrm{GHz}$ in the whole plasma volume. Thus, it can be expected that in the experiments described here the condition $f_{c e}>f_{p e}$ is always satisfied with $\mathrm{n}_{\mathrm{e}}<10^{12} \mathrm{~cm}^{-3}$. 
The range of measured emission frequencies $(9-12.5 \mathrm{GHz})$ during the instability corresponds to several scenarios of wave modes with right-hand polarization and effective interaction with the electrons.

Quasi-transversal (with respect to external B-field) fast (X-) or slow (Z-) extraordinary modes can be excited in rarefied plasmas [16,30] when $f_{p e} / f_{c e}<\beta=\left\langle v_{\text {hot }}\right\rangle / c$ where $\left\langle v_{\text {hot }}\right\rangle$ is the average velocity of the hot electrons interacting with the excited wave and $\mathrm{c}$ is the (vacuum) speed of light. Such transverse modes are excluded at the initial stage of the afterglow $(<10$ ms) because in our experiment $f_{p e} / f_{c e}$ is not significantly less than $0.2-0.5$, which is the approximate range of $\beta$ (10-60 keV electrons). Furthermore, the $\mathrm{X}$-mode waves are excited at frequencies $f>f_{c e}+f_{p e}{ }^{2} / f_{c e}$ which is in contradiction with the observed emission below the minimum electron cyclotron frequency of cold electrons. Another argument against the aforementioned transverse modes comes from the falling tones of the dynamic spectrum of the microwave bursts. The discussed condition for right-hand polarized extraordinary mode should be first fulfilled for more energetic electrons corresponding to lower (relativistic) electron cyclotron frequency and, thus should result to rising frequency tones as $f=f_{c e} \sqrt{1-\beta^{2}}$. Hence, only quasi-longitudinal modes will be considered hereafter.

Figure 7 shows the refraction index for waves with quasi-longitudinal $\left(\mathrm{k}^{\wedge} \mathrm{B}=5^{\circ}\right)$ propagation for $f_{p e}=6 \mathrm{Ghz}\left(\mathrm{n}_{\mathrm{e}}=4.4 \times 10^{11} \mathrm{~cm}^{-3}\right.$, which corresponds roughly to the assumed plasma density at the moment of the first instability event) and $\mathrm{f}_{\mathrm{ce}}=14 \mathrm{GHz}$ (which corresponds to the assumed spatial location of the maximum anisotropy of the hot electrons, i.e. $\mathrm{B}=\mathrm{B}_{\mathrm{ECR}}$ ). The following conclusions are not sensitive to the choice of specific value of the plasma density, which has been used for illustration purposes. The whistler branch of the ordinary mode can be excited at frequencies $f<f_{p e}<f_{c e}$, while the most effective excitation occurs near plasma resonance $\left(f=f_{p e}\right)$. Thus, the frequency of whistlers should strongly depend on the plasma density [31], which is affected by microwave power and neutral gas density (confirmed by the total extracted current). However, the plasma emission characteristics (frequencies) were experimentally demonstrated to be independent of the plasma parameters and, thus, the excitation of whistlers is considered improbable. Additional evidence against whistlers comes from fast sweeping tones of the dynamic spectrum of electromagnetic signal: the frequency drops by $1-2 \mathrm{GHz}$ during some microseconds which is too rapid to be explained by plasma density variations during the decay.

The most probable excited wave is the slow extraordinary Z-mode propagating quasilongitudinally with respect to the external magnetic field. Such waves are excited with frequencies of $f_{p e}<f<f_{c e}$, which matches the experimental conditions. In comparison to whistlers the refractive index of this mode does not approach infinity at $f_{\text {pe }}$ which allows the wave to propagate through $f=f_{p e}$ from the dense plasma into vacuum. The dependence of the group velocity of the quasi-longitudinal mode on the plasma density is presented in Fig. 8. The group velocity $V g$ decreases with the growth of the plasma density, and becomes significantly less than the speed of light in vacuum. This remarkable property favors effective amplification of parallel Z-mode, in comparison to for example the transverse Z-mode whose group velocity is close to $c$ in rarefied plasma, since the interaction time with resonant electrons is greatly increased. Thus the averaged exponential growth rate: $G=\int_{-L / 2}^{L / 2} \frac{\gamma_{z} d z}{V_{g(z)}} \cong \frac{\bar{\gamma} L}{V_{g}}(\gamma$ is the linear instability growth rate and $L$ is the length of interaction 
path), which determines the total amplification of the wave on the interaction path, can become rather high even at small values of $\gamma$. Moreover, at high plasma density even during one pass through the interaction region the wave can gain enough energy to expel energetic electrons from the trap. In other words, for quasi-longitudinal Z-mode in dense plasma there is no need for multi pass propagation in a resonator-like structure, which is quite typical for transverse modes [13] and whistlers [31].

A detailed study of the growth rates and frequency spectrum of cyclotron instability of Zmode at small propagation angles was performed in Ref. [30]. The maximum growth rate of the longitudinal Z-mode is:

$$
\gamma_{\max }=\frac{f_{p e, h o t}^{2}}{f_{c e}} 2 \sqrt{\pi} y^{\frac{3}{2}}(2 b y-3) \exp (-y)
$$

where $f_{p e, h o t}$ is plasma frequency of hot electrons, $b=1-\frac{\beta_{h}^{2} f_{p e}^{2}}{f_{c e}^{2}}, \beta_{h}^{2}=\frac{E_{h o t}}{m c^{2}}, E_{h o t}-$ is the energy of hot electrons, and $y$-is derived from following expression determining the optimal emission frequency $f_{\text {opt }}$, which corresponds to the maximum growth rate:

$$
y \equiv \frac{2}{\beta_{h}^{2}}\left(\frac{f_{c e}}{f_{o p t}}-1\right)=\frac{5 b+3}{4 b}+\sqrt{\left(\frac{5 b+3}{4 b}\right)^{2}-\frac{9}{4 b}}
$$

These formulas were obtained for loss-cone distribution of hot electrons at the limit of maximum anisotropy, and thus can be applied for qualitative estimates of optimal frequency and maximum growth rate of cyclotron instability in the ECRIS plasma. The dependencies of $f_{\text {opt }}$ on the plasma density at different electron cyclotron frequencies $\left(f_{c e}=10,12,14 \mathrm{GHz}\right)$ for a fixed energy of hot electrons $\left(E_{\text {hot }}=30 \mathrm{keV}\right)$ and on the electron cyclotron frequency for a fixed plasma frequency $\left(f_{p e}=9 \mathrm{GHz}\right)$ for three different values of hot electron energy $\left(E_{\text {hot }}=\right.$ $30,60,90 \mathrm{keV}$ ) are presented in figures 9,10 respectively. Optimal emission frequency $f_{\text {opt }}$ depends only weakly on the plasma density $\left(f_{p e}\right)$, but is mostly affected by the local electron cyclotron frequency and the average energy of the hot electron population interacting with the wave. It is clearly seen from the figures that $f_{\text {opt }}$ decreases both with increasing hot electron energy, and by decrease of the magnetic field $\left(f_{c e}\right)$.

The range of the plasma density for the most effective amplification of the quasi-longitudinal slow extraordinary wave is limited by the polarization properties. At high plasma density, i.e. $f_{\text {opt }}<f_{p e}$, the interaction with electrons is weakened due to left handed polarization of the wave [32]. At low plasma density or higher electron energies, i.e. $f_{p e} / f_{c e}<\beta$, the amplification of longitudinal waves is not effective due to relativistic effects, and the excitation of transverse extraordinary modes is favored [30].

Based on the listed (basic) properties of the quasi-longitudinal z-mode a scenario of cyclotron instability in decaying plasma, which explains the main features of the electromagnetic emission spectrum, can be deduced. Let us assume that during the stochastic heating stage of the ECR discharge the hot electron population is distributed axially between the center of the trap and the ECR surface. Since the maximum anisotropy is achieved near the local reflection point of the bouncing electrons $\left(\mathrm{v}_{\|}=0\right.$ at the mirror point), it can be argued that the most effective interaction is initiated near the cold electron cyclotron frequency $f_{c e}=f_{\mu w}=14 \mathrm{GHz}$. However, the conditions for z-mode wave excitation are not fulfilled immediately after switching off the microwave power as the plasma density should decay enough to reach 
$f>f_{p e}$ for right handed polarization. We suppose that the reason for the delay is related to rather high hot electron energy, which causes the optimum emission frequency to shift lower. Thus, the moment of appearance of first electromagnetic pulse is determined by the characteristic lifetime of cold (warm) electron component.

The wave begins to propagate towards descending magnetic field and is amplified by hot electrons distributed between the ECR zone and the center of magnetic trap. The decrease of the local electron gyrofrequency results to the falling spectral tone of the electromagnetic emission. The non-linear dynamics of the wave particle interaction can be qualitatively described by the following equations for the hot electron density $N_{h}$ and the electromagnetic energy density $E_{w}[13]$ :

$$
\left\{\begin{array}{l}
\frac{d N_{h}}{d t}=-\kappa E_{w} N_{h} \\
\frac{d E_{w}}{d t}=h E_{w} N_{h}-v E_{w}
\end{array}\right.
$$

The first equation describes the losses of hot electrons induced by the high-frequency field (the $\kappa$ - parameter characterizes the particle losses) and the second equation describes the field growth or decay, depending on the growth $\left(h=\gamma / N_{h}\right)$ and damping $\left(v=v_{e i}\right.$ - electron collision) rates. Using equation (1) for the range of plasma frequencies $f_{p e}=6-9 \mathrm{GHz}, f_{c e}=$ $14 \mathrm{GHz}, E_{h}=30 \mathrm{keV}$ gives estimated maximum growth rate of (0.07-0.09) $N_{h}$. Taking the density of hot electron population of about $\sim 10^{8}-10^{9} \mathrm{~cm}^{-3}$ will result in $\gamma_{\max } \approx$ $10^{7} \div 10^{8} S^{-1}$, which is the same order with the estimation of growth rate roughly deduced from the experimentally observed pulse duration $\left(\gamma_{0} \sim \tau^{-1}, \tau \approx 10-1000 n s\right)$ at the initial (linear: $\left.E \sim e^{\gamma t}\right)$ stage of the cyclotron instability. Thus the growth rate significantly exceeds the damping rate $\left(v \sim 10^{2} s^{-1}\right)$. As soon as the instability growth rate is proportional to the density of hot electrons (see formula (1)) the dynamics of the instability will be determined solely by the losses of hot electrons into the loss-cone. In other words, if the conditions for the excitation of quasi-longitudinal Z-mode are fulfilled $\left(f_{\text {opt }}>f_{p e}\right)$, the instability will last until the damping rate exceeds the growth rate.

Besides the hot electron losses the electromagnetic pulse can stop due to modifications of the polarization properties of the wave. This interpretation is supported by the fact that the decrease of the emission frequency is much faster than the decay of the warm plasma component, i.e. the system can go below the threshold due to left-handed polarization of the extraordinary mode at $f_{\text {opt }}<f_{p e}$. The (down)shift of the emission frequency is caused by decreasing of local relativistic electron cyclotron frequency, which can occur due to the discussed shifting of the interaction region to lower magnetic field. Another possible option is related with the reduction of $f_{c e}$ due to relativistic effects for hot electrons with higher energies. The maximum growth rate is realized for the hot electrons with moderately low energies [30], which are first precipitated from the trap during the instability, and thereby increasing of the mean energy of the remaining hot electrons.

As soon as the first electromagnetic pulse is emitted and a certain fraction of the hot electrons has left the trap, the system becomes stable until the plasma decays enough to again fulfil the condition $f_{\text {opt }}>f_{\text {pe }}$. The delay between the first and the second electromagnetic pulse depends on the characteristic time of the plasma decay. The generation of the next pulse of Z-mode 
starts at the spatial location where the hot electrons have remained, thus shifting the interaction region towards the trap center. The generation of pulse sequences will repeat until all hot electrons have left the trap and the emitted frequency spectrum will cover the whole bandwidth, which corresponds to interaction region between ECR heating zone and the trap center. It is worth to be mentioned, that each subsequent burst of electromagnetic emission occurs at lower plasma density, meaning higher group velocity of slow extraordinary wave (see Fig. 8). The interaction with the electrons becomes faster and results in steeper slope of emission spectrum from pulse to pulse (see fig. 4a-c): i.e. the frequency of the last pulse in a sequence drops as fast as 1-2 GHz in 30-70 ns. These dispersion properties also explain the shape of the spectrum depicted in Fig. 11, which was recorded $20 \mu$ s before switching off the klystron. The gentle slope of the spectrum exhibits a dominant frequency of $12.6 \mathrm{GHz}$ and corresponds to the generation of quasi-longitudinal extraordinary mode with very low group velocity. This regime of cyclotron instability was already investigated in sustained plasma [33] and possibly is a particular case of the discussed mechanism in dense plasma.

At latter stage of plasma decay (>10 ms) the plasma density can become so low that the condition for excitation of the transverse Z-mode $\left(f_{p e} / f_{c e}<\beta \beta\right)$ can be fulfilled for hot electrons. These changes in the scenario of cyclotron instability can be clearly seen from the dynamic spectrum of the last electromagnetic burst (see fig. 5): the rapid falling tone is replaced by generation of narrow-banded emission. This regime is concerned with interaction of transverse $\mathrm{z}$-mode with hot electrons located at the trap center and is quite typical for rarefied plasma $[13,14]$

\section{Conclusion}

The proposed mechanism of cyclotron instability allows explaining the basic properties of the dynamic spectra of the observed microwave emission in the decaying plasma of a minimumB ECRIS. The analysis has shown that the instability is driven by the resonant interaction of hot electrons, distributed between the ECR zone and the trap center, with slow extraordinary wave, which propagates quasi-parallel to the magnetic field. The characteristic timescales of the instability, such as the delay after switching off the microwave power and the repetition period between pulses, are dictated by the rate of the plasma decay. The slope of the frequency spectrum during the pulse depends on the absolute value of the plasma density, which determines the group velocity of the Z-mode. The falling frequency tones of each pulse as well as the total downward trend of the whole spectrum during the decay are associated to the decrease of the local relativistic cyclotron frequency. The research results can be claimed not only to improve the understanding of modern ion sources, but also advance studies of wave-particle interactions in non-equilibrium plasma.

\section{Acknowledgements}

This work has been supported by the Academy of Finland under the Finnish Center of Excellence Program 2012-2017 (Nuclear and Accelerator Based Physics Research at JYFL) and researcher mobility grants 267174 and 267227. Work of Ivan Izotov was supported by the fellowship of the President of Russian Federation for young scientists \#SP-7279.2013.2. 
Work of Dmitry Mansfeld was partially supported by Russian Foundation for Basic Research \# 15-32-20770, and by the Ministry of Education and Science of Russian Federation (project on. 14.Z50.31.0007). Work was supported by the grant of the President of Russian Federation for young scientists \# MK-6565.2014.2The authors thank Tektronix Inc. for technical support.

\section{References}

[1] A Köhn, G Birkenmeier, E Holzhauer, M Ramisch and U Stroth 2010 Plasma Phys. Control. Fusion 52035003

[2] P.A. Bagryansky et al 2015 Nucl. Fusion 55053009

[3] W Lee, G S Yun, H K Park, Y B Nam and C R Seon 2014 Plasma Phys. Control. Fusion 56095017

[4] R. C. Garner, a M. E. Mauel, b S. A. Hokin, R. S. Post, and D. L. Smatlak 1987 Phys. Rev. Lett. 591821

[5] Tarvainen O et al 2014 Plasma Sources Sci. Technol. 23025020

[6] K Langbein 1996 Rev. Sci. Instrum. V. 67, Issue 3, pp. 1334-1336

[7] Maunoury L et al 2008 Rev. Sci. Instrum.79(2 Pt 2):02A313

[8] L. Dumas, C. Hill, D. Hitz, D. Kuchler, C. Mastrostefano, M. O’Neil, R. Scrivens. 2007 High Energy Physics and Nuclear Physics; v. 31; p. 51-54

[9] R.C. Garner, M.E. Mauel, S.A. Hokin, R.S. Post, and D.L. Smatlak. 1990 Phys. Fluids B 2,242

[10] Izotov I, Mansfeld D, Skalyga V, Zorin V, Grahn T, Kalvas T, Koivisto H, Komppula J, Peura P and Tarvainen O 2012 Phys. Plasmas 19122501

[11] Gurnett D A 1974 Geophys J. Res. 7928

[12] Panchenko M, Rucker H O and Farrell W M 2013 Planet. Space Sci. 77 3-11

[13] A.V. Vodopyanov, S.V. Golubev, A.G. Demekhov, V.G. Zorin, D.A. Mansfeld, S.V. Razin, A.G. Shalashov. 2007 Journal of Experimental and Theoretical Physics V.104(2), P. 296-306. 
[14] M.E. Viktorov, A.V. Vodopyanov, S.V. Golubev, I.V. Izotov, D.A. Mansfeld 2013 Radiophysics and Quantum Electronics. V.56. №1. P.12-19.

[15] A.G. Shalashov, A.V. Vodopyanov, S.V. Golubev, A.G. Demekhov, V.G. Zorin, D.A. Mansfeld, S.V. Razin. 2006 JETP letters, V.84 (6), P. 314-319

[16] S. V. Golubev and A. G. Shalashov 2007 Phys. Rev. Lett. Vol. 99 , P. 205002(4).

[17] A.G. Shalashov, S.V. Golubev, E.D. Gospodchikov, D.A. Mansfeld and M.E. Viktorov. 2012 Plasma Phys. Control. Fusion, V.54, N.8, P.085023.

[18] V. V. Alikaev, V. M. Glagolev, S. A. Morosov 1968 Plasma Phys. 10 (8), 753.

[19] M Viktorov, D Mansfeld, S Golubev 2015 Europhysics Letters. 109 p.65002.

[20] Koivisto H, Heikkinen P, Hänninen V, Lassila A, Leinonen H, Nieminen V, Pakarinen J, Ranttila K, Ärje J and Liukkonen E 2001 Nucl. Instrum. Methods B 174379

[21] Tarvainen O, Kalvas T, Koivisto H, Ropponen T, Toivanen V, Vainionpää J H, Virtanen A and Ärje J 2009 Proc. HIAT (Venice, Italy, 8-12 June) http://JACoW.org

[22] Barue C, Lamoreux M, Briand P, Girard A and Melin G 1994 J. Appl. Phys. 765

[23] Douysset G, Khodja H, Girard A and Briand J P 2000 Phys. Rev. E 613

[24] Tarvainen O et al 2015 Plasma Sources Sci. Technol. 24035014

[25] Girard A, Hitz D, Melin G and Serebrennikov K 2004 Rev.Sci. Instrum. 751381

[26] Mironov V and Beijers J P M 2009 Phys. Rev. ST Accel. Beams 12073501

[27] T. Ropponen, O. Tarvainen, P. Jones, P. Peura, T. Kalvas, P. Suominen and H. Koivisto, 2009 Nuclear Instruments and Methods in Physics Research, Section A, Volume 600 , Issue 3, p. 525-533

[28] O. Tarvainen, T. Ropponen, V. Toivanen, T. Kalvas, J. Ärje and H. Koivisto, 2010 Plasma Sources Sci. Technol. 1904502.

[29] Lamy, L., B. Cecconi, P. Zarka, P. Canu, P. Schippers, W. S. Kurth, R. L. Mutel, D. A. Gurnett, D. Menietti, and P. Louarn 2011 J. Geophys. Res., 116, A04212

[30] Demekhov A G 1987 Radiophys. Quantum Electron. 30 547-57 
[31] Trakhtengerts V Yu and Rycroft M J 2008 Whistler and Alfvén Mode Cyclotron Masers in Space (Cambridge: Cambridge University Press) p 36

[32] Ginzburg V L 1970 The Propagation of Electromagnetic Waves in Plasmas (Oxford: Pergamon)

[33] I Izotov, O Tarvainen, D Mansfeld, V Skalyga, H Koivisto, T Kalvas, J Komppula, R Kronholm, J Laulainen. 2015 Plasma Sources Science and Technology. 24045017. 


\section{List of figures}

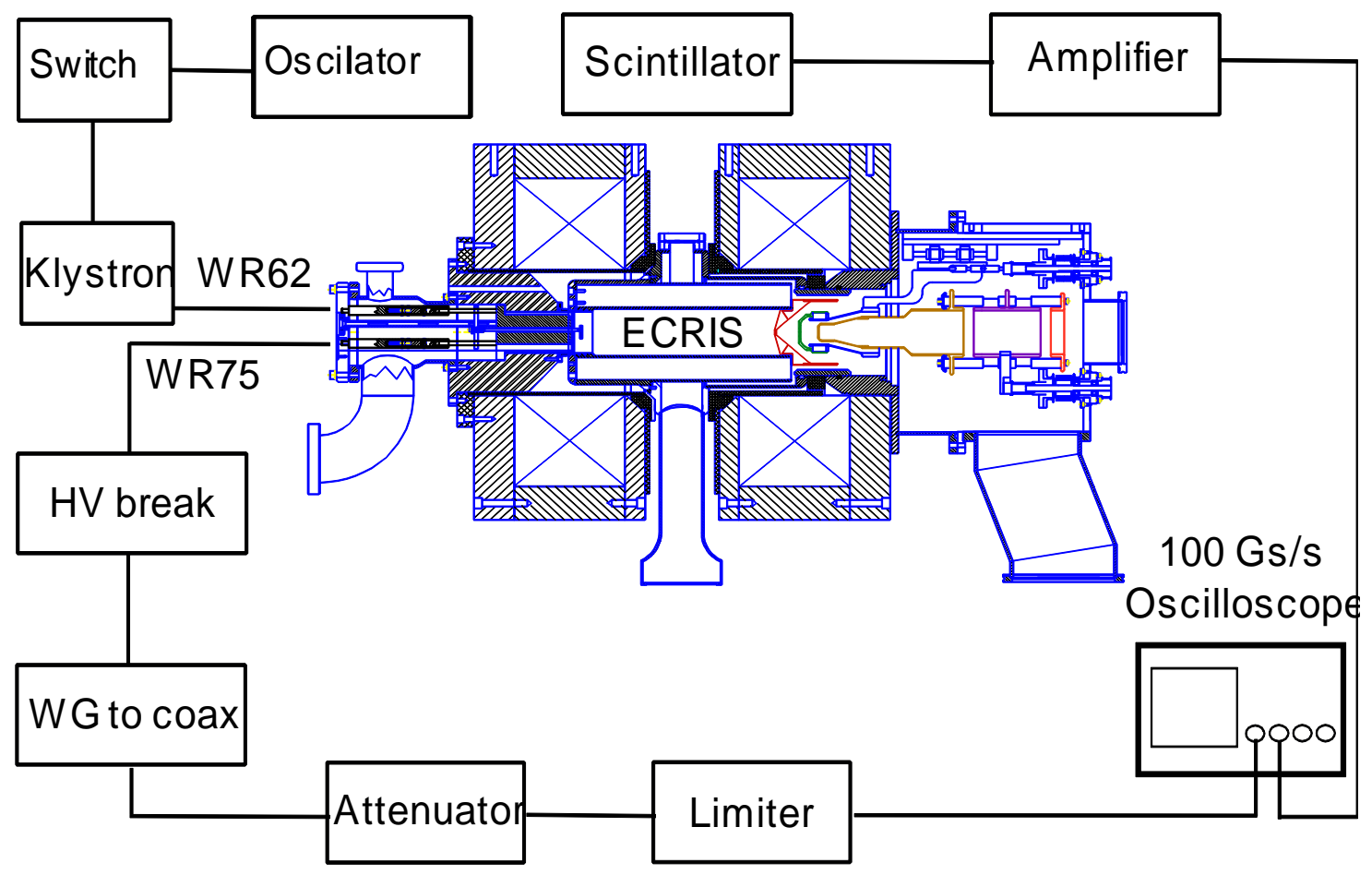

Figure 1. Schematic figure of the experimental setup (left) and injection iron plug of the JYFL $14 \mathrm{GHz}$ ECRIS viewed from the extraction (right). The primary microwave port (WR62) used for $14.085 \mathrm{GHz}$ injection and the diagnostics port (WR75) are indicated in the figure. 


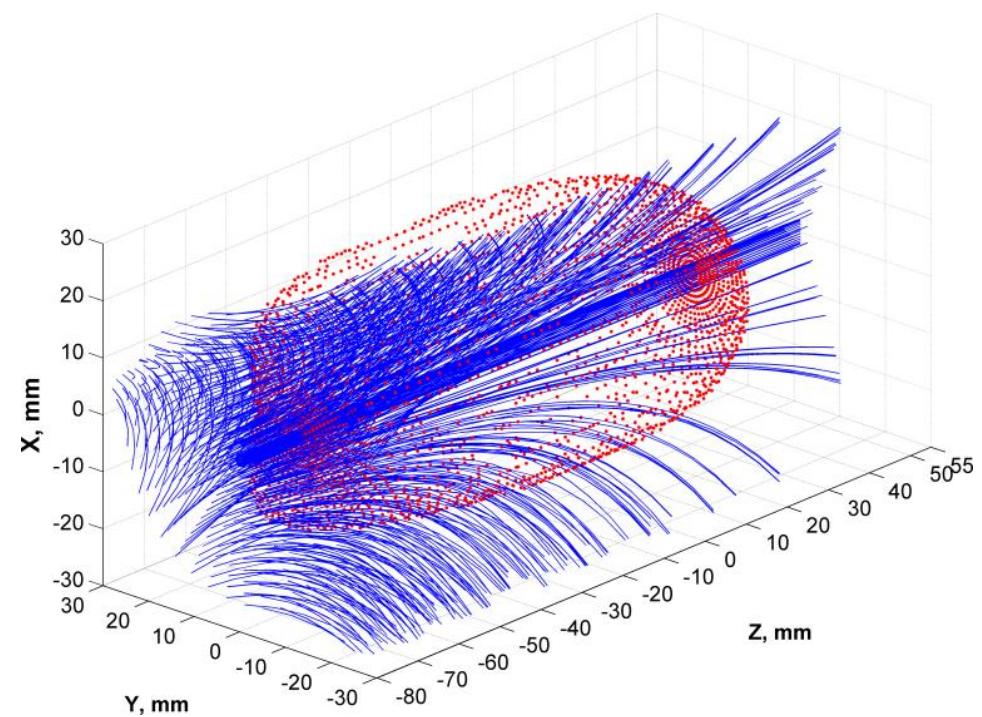

Figure 2a. 3D plot of the magnetic field lines of JYFL $14 \mathrm{GHz}$ ECRIS. Red dots are depicting the place of resonance surface.

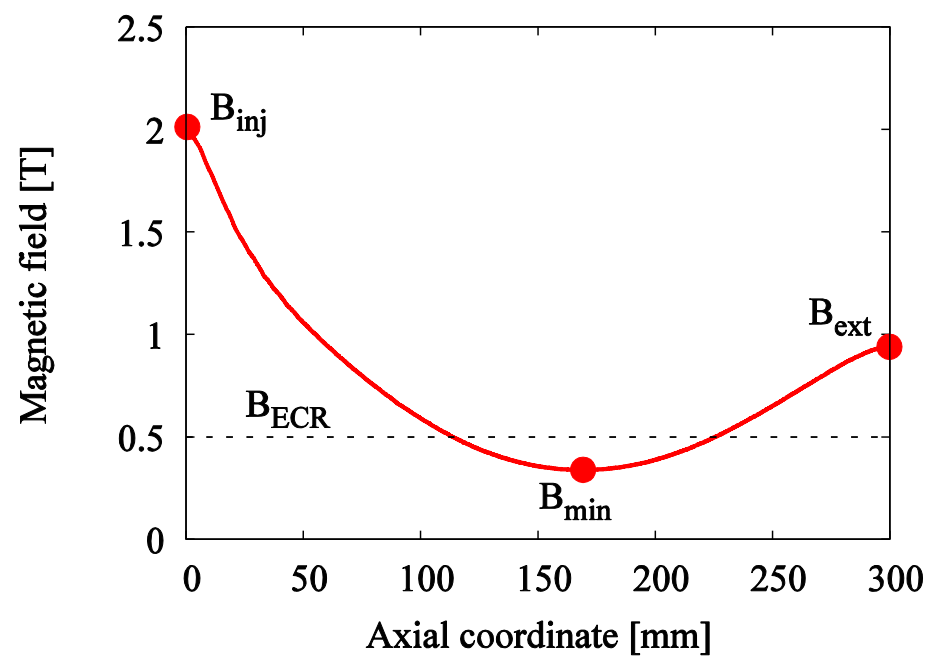

Figure 2b. An example of the magnetic field strength on axis . Binj Bext, Bmin and BECR are corresponding magnetic field strengths for injection, extraction, B-minimum and resonance $(14 \mathrm{GHz})$. The field strength is affected by the solenoid coil currents while the topology remains the same.. 

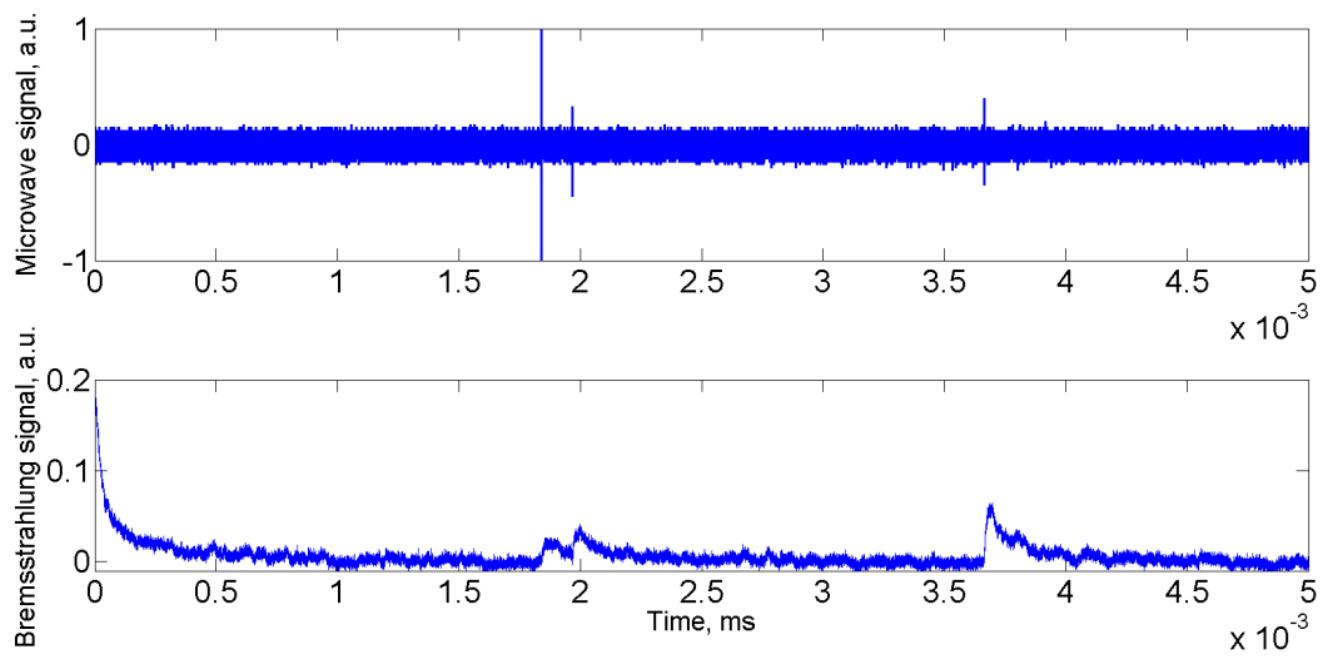

Figure 3. Typical waveforms of the microwave emission and bremsstrahlung signals during a single onset of the cyclotron instability. Microwave heating is switched off at $t=0$. The data is acquired with $600 \mathrm{~W}$ of injected microwave power at $2.1 \cdot 10^{-7}$ mbar of argon and $\mathrm{B}_{\min } / \mathrm{B}_{\mathrm{ECR}}=0.75$ 

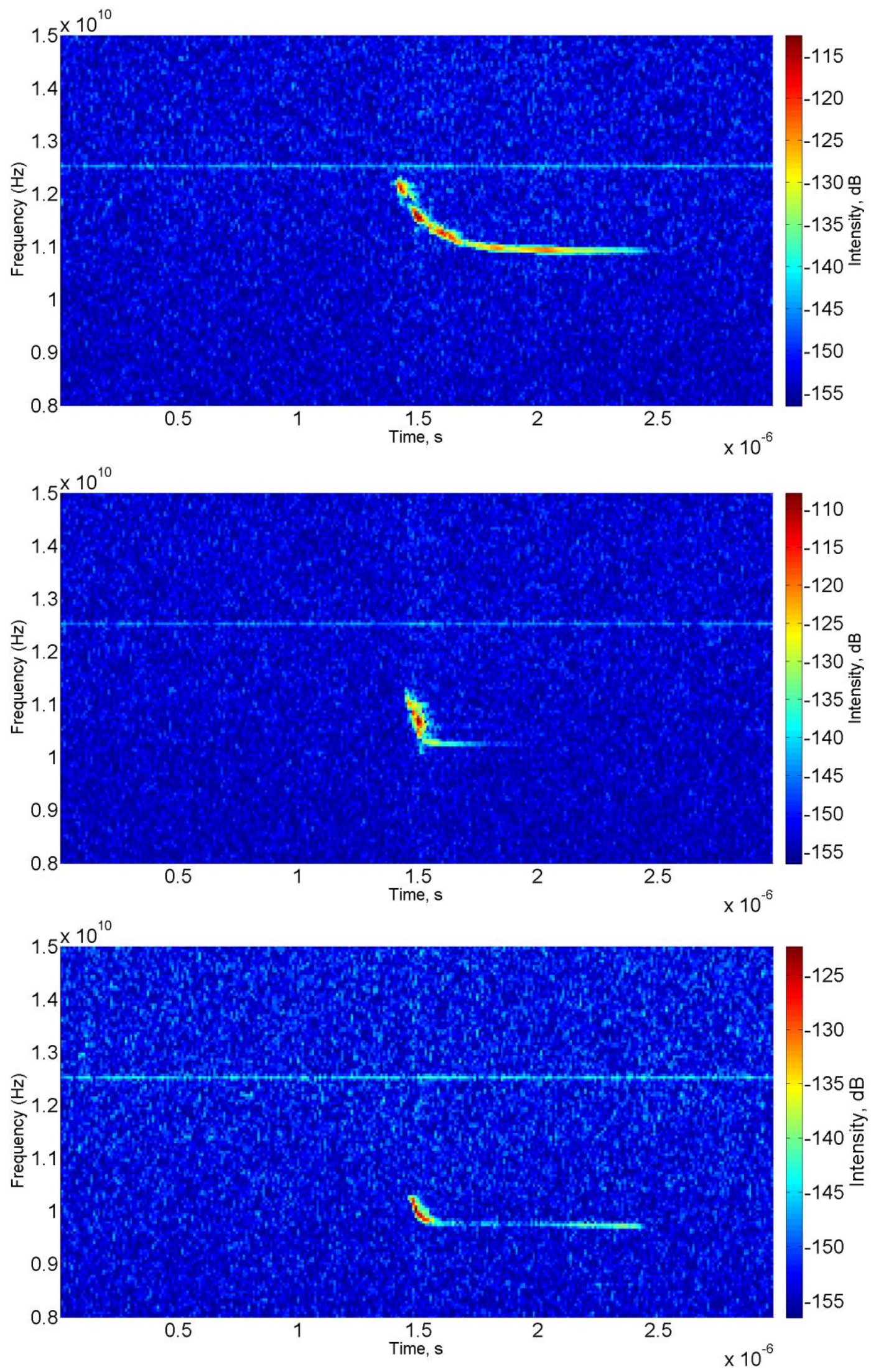

Figures 4a-4c Dynamic spectrograms of three instability microwave pulses 


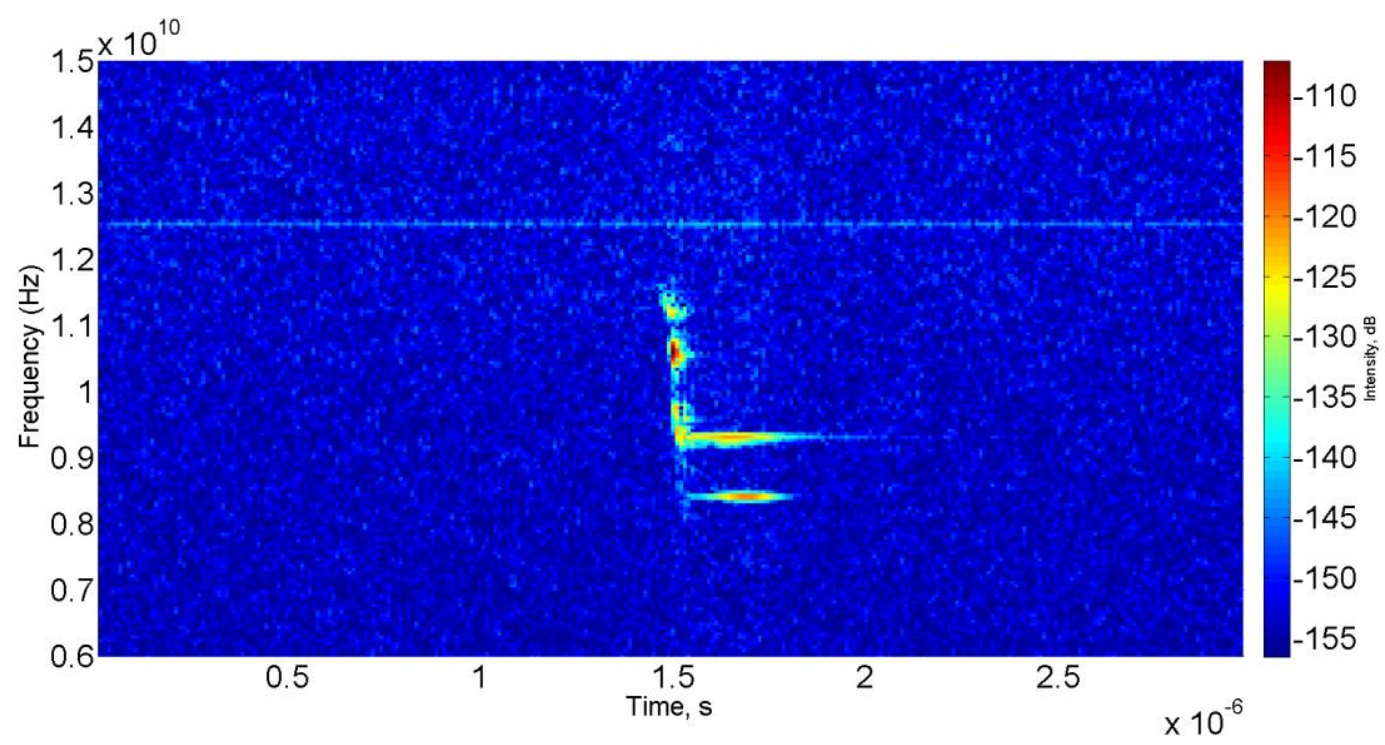

Figure 5. Example of quasi-monochromatic narrow-banded harmonics in the dynamic spectrogram of the microwave signal.
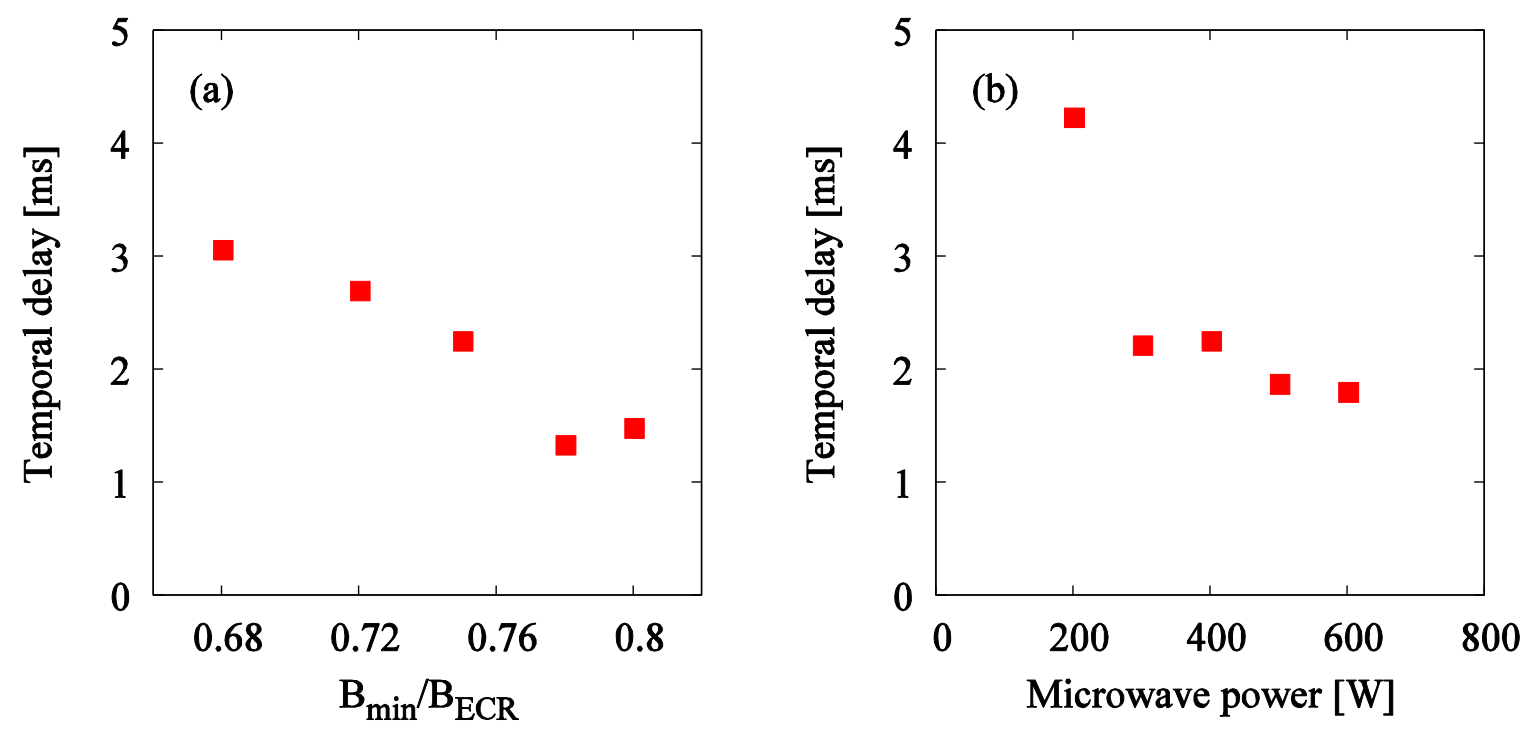

Figures 6a and 6b. Dependence of temporal delay (time between switching off the microwave heating and appearance of the first instability pulse in decaying plasma) on magnetic field (a) and power (b) 


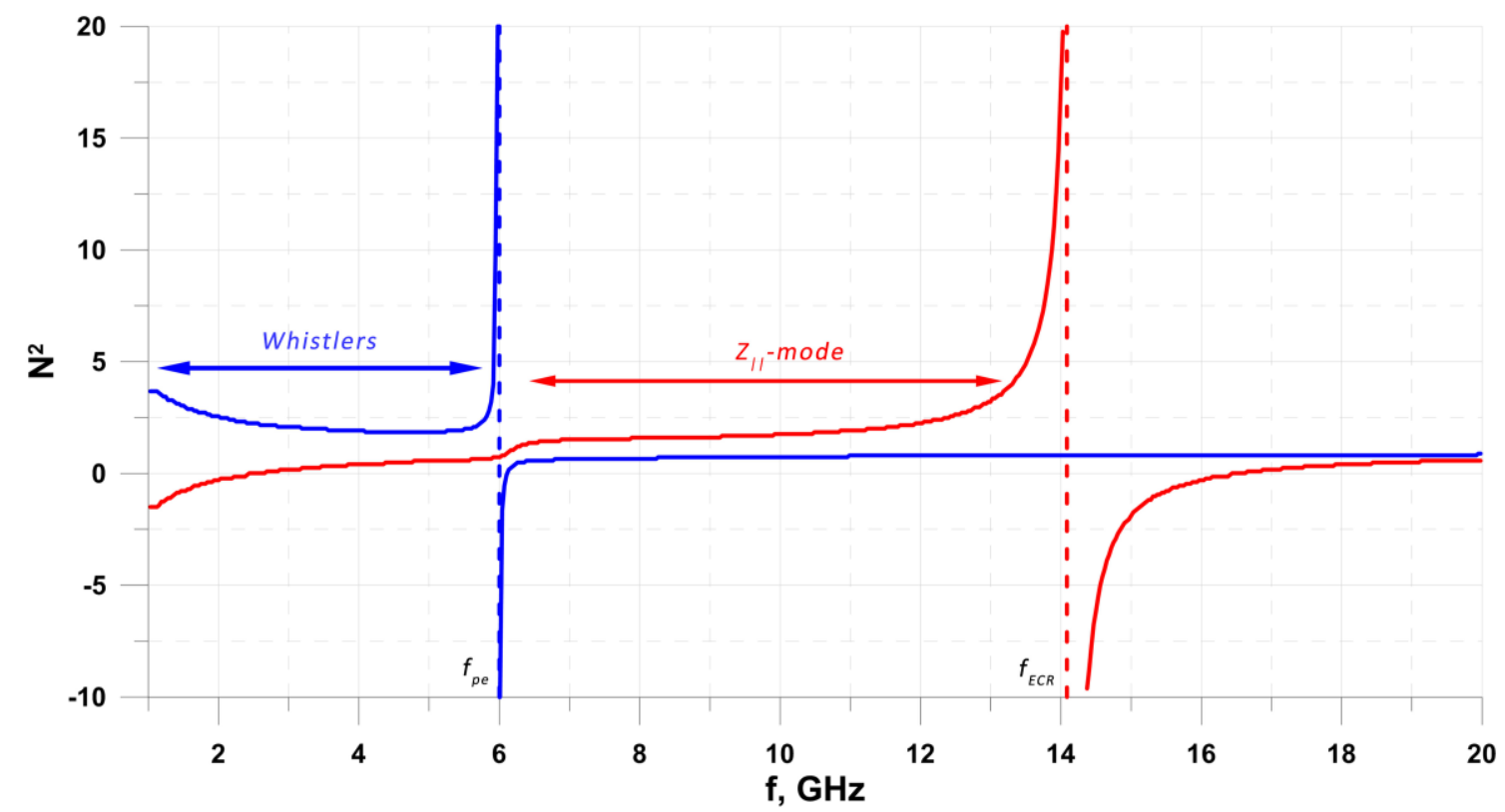

Figure 7. Refractive index of longitudinally propagating electromagnetic waves in plasma, $f_{p e}$ $=6 \mathrm{GHz}, f_{c e}=14 \mathrm{GHz}$. Ordinary (blue curve) and extraordinary (red curve) waves.

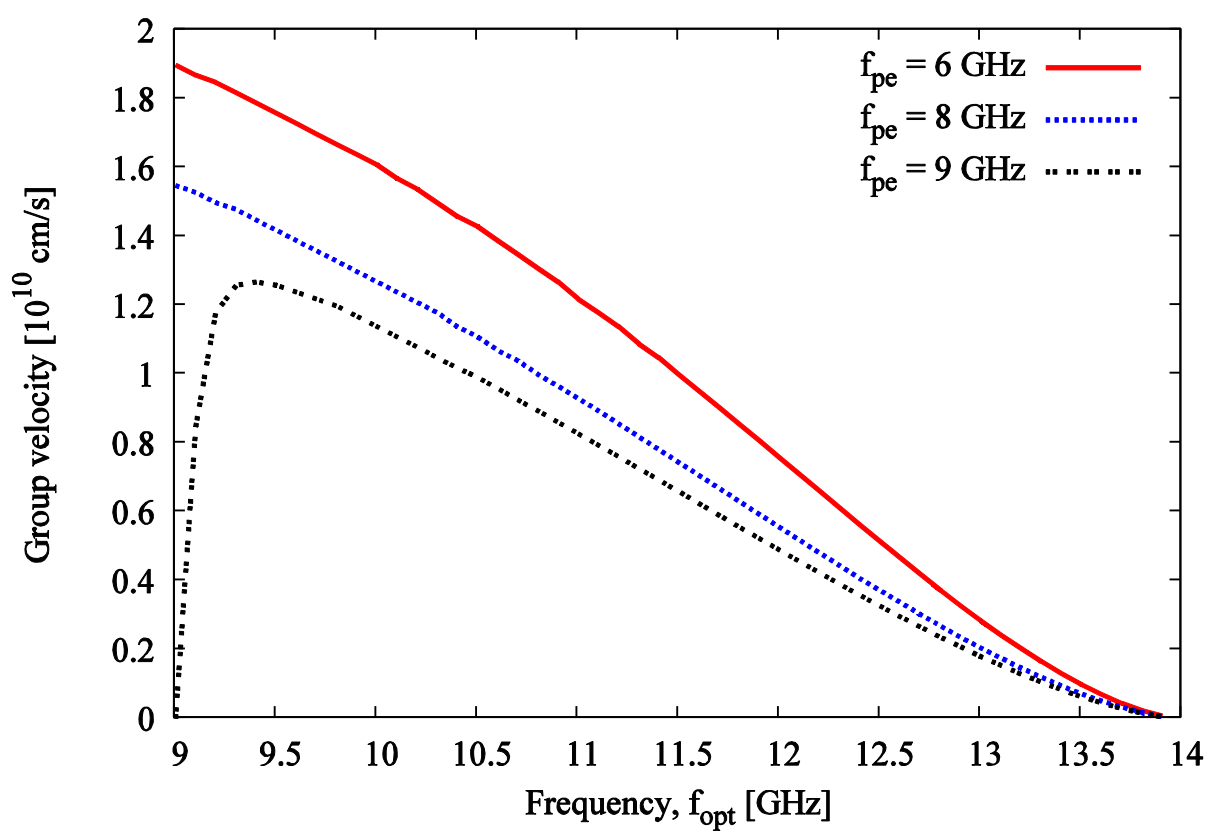

Figure 8. Group velocity of quasi-longitudinal Z-mode versus optimal frequency $f_{\text {opt }}$ at different values of plasma frequency $f_{p e}$. 


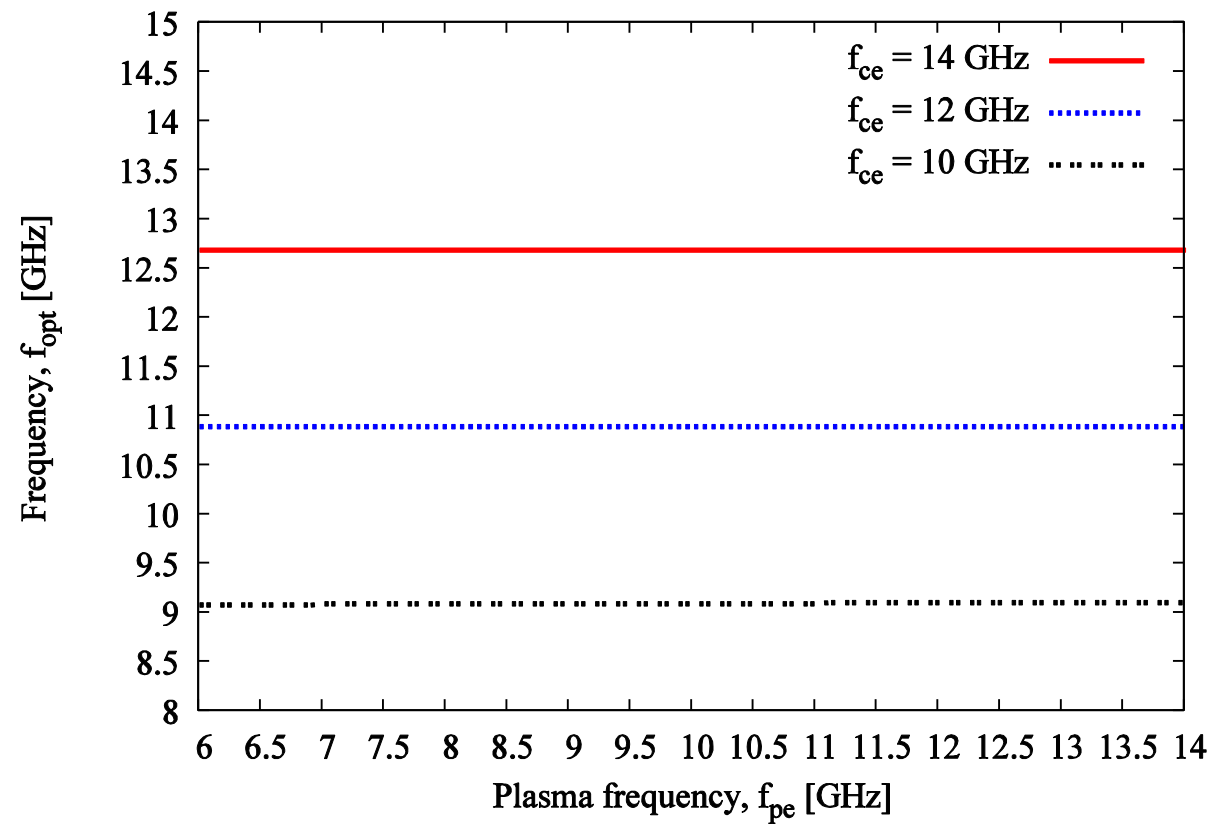

Figure 9. Optimal emission frequency $f_{\text {opt }}$ versus plasma frequency $f_{p e}$ at different cyclotron frequencies and fixed energy of hot electrons $E_{\text {hot }}=30 \mathrm{keV}$.

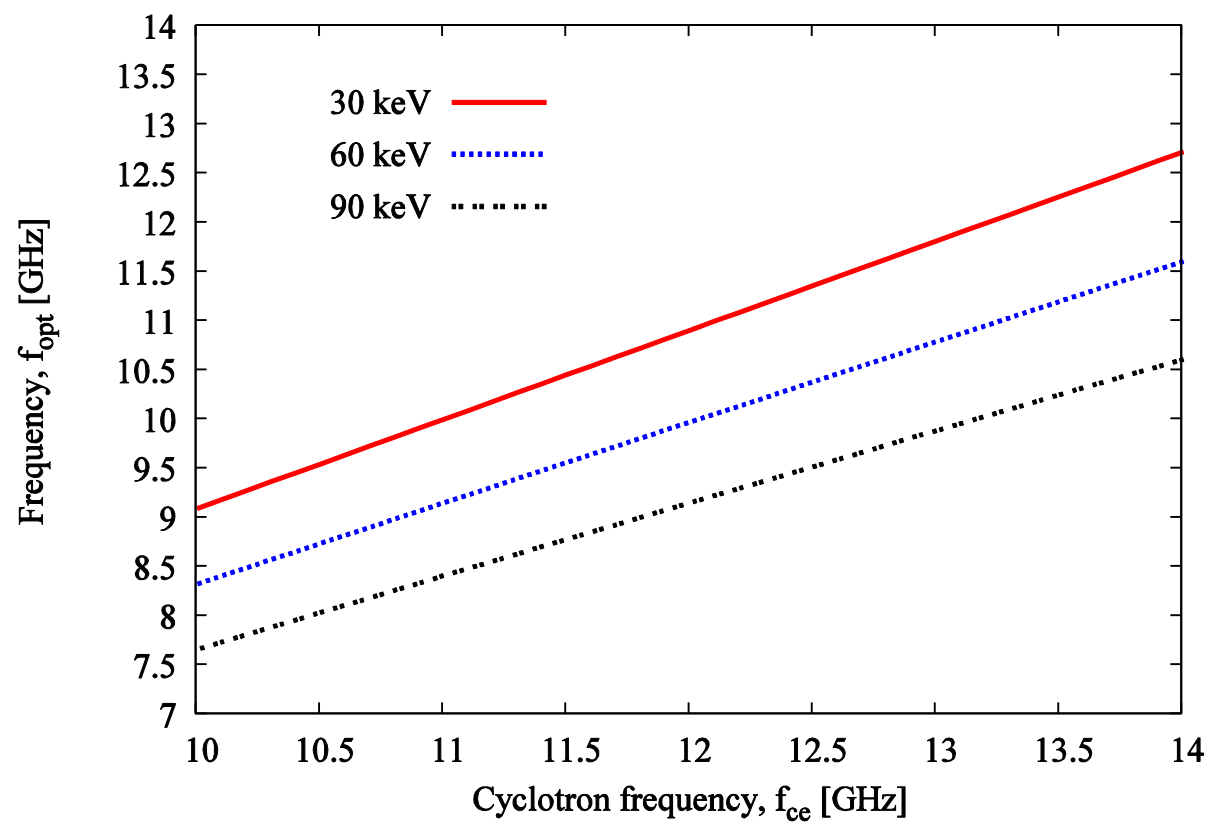

Figure 10. Optimal emission frequency $f_{\text {opt }}$ versus cyclotron frequency $f_{c e}$ at different energies of hot electrons $E_{\text {hot }}$ and fixed plasma frequency $f_{p e}=9 \mathrm{GHz}$. 


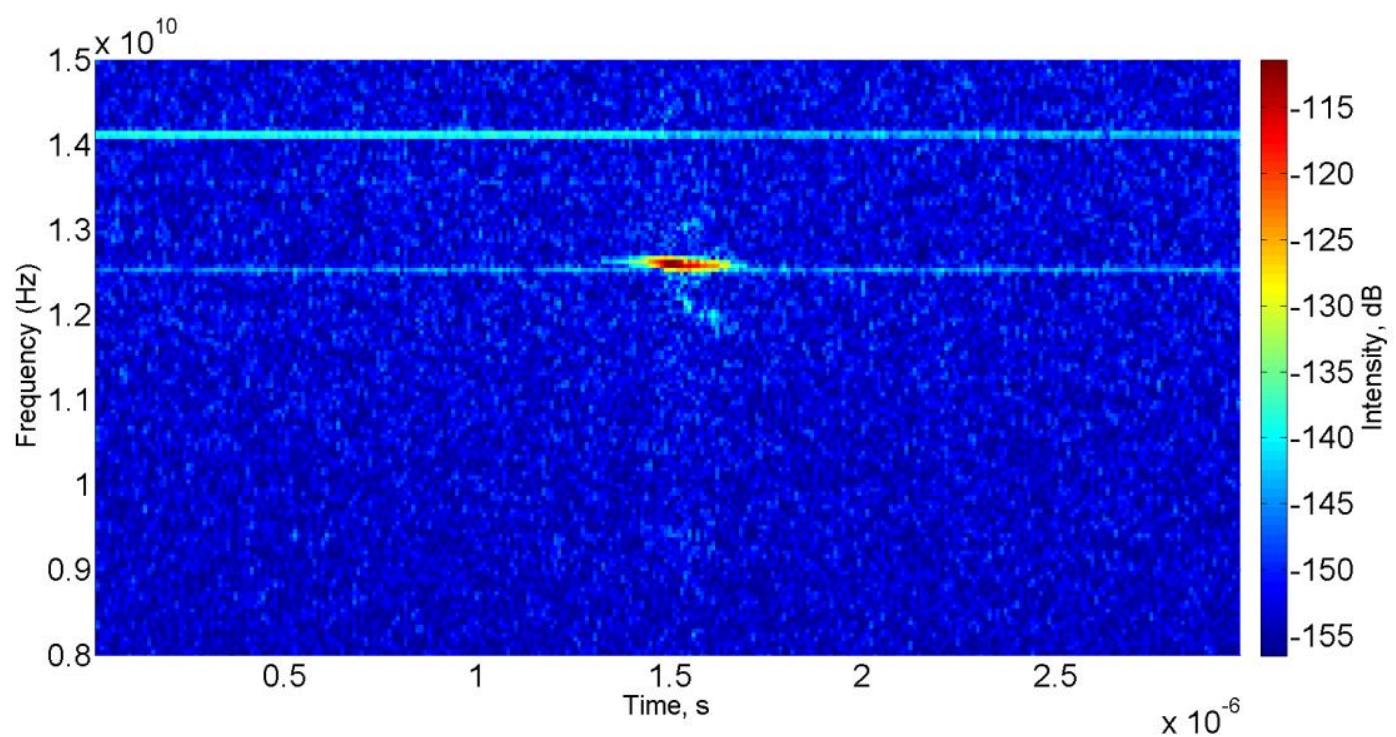

Figure 11. Dynamic spectrogram of the microwave signal before switching off the klystron. 